\title{
Implementación de procuramiento de plaqueta ósea para cranoplastia en pacientes neuroquirúrgicos en hospital San Pablo de Coquimbo
}

\section{Implementation of bone flap cranioplasty handling process in neurosurgical patients at San Pablo Hospital, Coquimbo}

\author{
Gilda Parra Fierro' \\ ${ }^{1}$ Neurocirujano Hospital San Pablo de Coquimbo. Coquimbo, Chile.
}

\begin{abstract}
Resumen
La craneoplastia para pacientes con cranectomia descompresiva es un procedimiento habitual en neurocirugía. EI material con el que se realiza la reparación es un tema controvertido, actualmente la alternativas para este procedimiento son a través de hueso autologo o injertos aloplástico. De las alternativas aloplásticas se encuentran los metales como el titanio, las cerámicas y los polímeros, estos últimos son los más utilizados y con mejor resultado. Considerando que el hueso autólogo, el cual requiere un sistema de almacenaje a través de criopreservación y a través de la esterilización con rayos gamma, es una alternativa efectiva y con evidencia científica de seguridad de su uso. Se describe en este trabajo como se implementa el procedimiento de procuramiento e implante de este tipo de plaqueta en un hospital de mediana complejidad (Hospital San Pablo de Coquimbo), discutiendo cada uno de los procesos definidos. Considerando la etapa desde la obtención del hueso hasta la reinstalación, con seguridad y cumpliendo estandares de seguridad. Dentro del analisis se determina los costos asociados al proceso de procuramiento e implante, comparandondola con las otras alternativas metálicas y de polímeros determinando el procuramiento de hueso autológo a través de la criopreservación como la de mayor costo efectividad.
\end{abstract}

Palabras clave: Craneoplastia, injerto autólogo, injerto aloplástico.

\begin{abstract}
The cranioplasty for patients with decompressive craniotomy is a standard procedure in neurosurgery. The materials that are used in the repairment operation is a controversial theme, currently the alternatives for this procedure are through autologous bone or alloplastic grafts. Within the alloplastic alternatives different metals can be found, for example titanium, ceramic, and polymers. These are the most commonly used and with the best results. Considering that the autologous bone, which requires a storage system through cryopreservation and through gamma ray sterilization, is an effective alternative with secure scientific evidence of its use. The work describes how the procurement procedure is implemented and implantation of platelet type in a medium complexity hospital (San Pablo hospital in Coquimbo), discussing each and every of the defined processes. Considering that from the stage of obtaining the bone to the reinstallation, with security and meeting security standards. Within the analysis, costs associated with the procurement and implant process are determined, comparing them to the other metallic and polymer alternatives determining the procurement of autologous bone through cryopreservation as the higher cost effectiveness.
\end{abstract}

Key words: Cranioplasty, autologous grafts, alloplastic grafts.

\section{Correspondencia a:}

gildaparrafierro@gmail.com 


\section{Introducción}

La cranectomia descompresiva es una opción terapéutica ampliamente aceptada para la hipertensión intracraneal refractaria secundaria a una lesión cerebral especialmente la lesión cerebral traumática ${ }^{1}$. Este procedimiento consiste en estos casos seleccionados en el retiro de hueso lo más grande posible, se ha recomendado que el tamaño de la descompresión para adultos debe ser como mínimo $14 \mathrm{~cm}$ en anteroposterior por $12 \mathrm{~cm}$ en superoinferior si la intención es realizar una descompresión frontotemporoparietal. Para realizar este procedimiento se deben tener consideraciones anatómicas importantes para respetar lesiones de senos venos y de la órbita de dicho lado².

Los pacientes que se someten a una craniectomía descompresiva, comunmente requieren una craneoplastia para proteger el cerebro, restaurar la estética, aliviar los sintomas neurologícos, complementar los defectos externos y ayudar a los pacientes a mejorar su autoestima ${ }^{3}$. Muchas publicaciones relacionan la craneoplastia con el mejor metabolismo cerebral de la glucosa, la reserva cerebrovascular, la regulación del flujo sanguineo postural y la circulación de líquido cefaloraquideo ${ }^{4,5}$.

Se recomienda la craneoplastia temprana (antes de las 6 semanas), pues se ha encontrado que mejora los resultados clínicos $^{6,7}$. En el aspecto de la infección postoperatotia, un estudio multinacional prospectivo diseñado dice que no existe diferencia entre las tasas de infección entre la craneoplastia temprana y la tardía?.

La craneoplastia, puede ser llevada a cabo a traves de diferentes materiales:

- Sustitutos óseos: El material sintético ideal debería ser fácil de moldear y ajustarse con precisión a los cráneos con un defecto craneal. El material lo suficientemente radiotransparente para evitar la formaciónn de srtefactos en las imágenes y biocompatibilidad para disminuir la respuesta inmune o la inflamación ${ }^{8,9}$.

Diversos materiales se utilizan como ateruiales sintéticos, como metales (titanio, etc), cerámica (fosfato de calcio, hidroxiapatita, etc) y polimeros (polimetacrilato de metilo, polietinelo poli-éter-éter-cetona, etc) ${ }^{10,11}$.

Recientemente se comercializan prótesis a medida para craneoplastia a medida para craneoplastia impresas con impresoras tridimensionales ${ }^{12}$.

- Colgajo de cráneo autólogo: Los huesos autólogos son económicos y cosmeticamente naturales ${ }^{10}$. Los colgajos óseos trasplantados se someten a complejos procesos de curación para fusionarse a los huesos circundantes ${ }^{13}$.

Los colgajos de cráneo frescos deben conservarse esterilmente después de la cranectomia descompresiva. Existe dos métodos para hacer que el colgajo de cráneo autólogo sea estéril para la craneoplastia: colocación de la plaqueta ósea bajo la capa subcutánea ya sea abdominal o subgaleal y crioconservación, si bien es cierto que no se han reportado tasas de complicaciones similares en ambos procedimientos, si dejar la plaqueta en región abdominal alarga la cirugía y evita la incisión abdominal ${ }^{14}$.

Actualmente, se recomienda la técnica de crioconserva- ción por ser el método más económico y práctico, congelando los injertos óseos entre -18 y $-83^{\circ} \mathrm{C}^{15}$. Estudios biomécanicos revelan que la congelación y la descongelación tienen poco efecto sobre las propiedades mecánicas del cráneo humano ${ }^{16}$. Actualmente, no se recomienda criopreservar las plaquetas por un tiempo mayor a 6 meses ${ }^{17}$.

Muchos investigadores enfatizan el mérito económico de la craneoplastia con colgajos óseos autólogos ${ }^{18}$. La diferencia de los resultados clínicos entre los materiles sintéticos y el injerto óseo autólogo en la craneoplastia es muy pequeña. Algunos informes dicen que el costo total acumulado, incluido los riesgos de complicaciones y los tratamientos, no fue significativamente diferente entre el colgajo de titanio y el hueso autologo en los Estados Unidos de América y Canadá ${ }^{19}$.

La evidencia actual recomienda los colgajos óseos criopreservados autólogos como el sustituto ideal para la craneoplastia, por sus capacidades biólogicas y por ser más económico ${ }^{20}$.

Actualmente, antes de la implementación del procuramiento de plaqueta ósea en el Servicio de Neurocirugía del hospital San Pablo de Coquimbo, se esta realizando craneoplastia con malla de titanio. En muy pocos pacientes se ha guardado la plaqueta en el tejido subcutáneo abdominal con reposición posterior de ella.

El objetivo del trabajo es describir el proceso de implementación de procuramiento y reposición de plaqueta ósea autóloga en pacientes neuroquirúrgicos cranectomizados, valorizar el proceso completo en pacientes en el hospital San Pablo de Coquimbo y describir los primeros pacientes operados bajo esa técnica.

\section{Desarrollo}

1. Proceso de implementación de procuramiento y reposición de plaqueta ósea autóloga en pacientes neuroquirúrgicos cranectomizados

- Establecer recursos con los que cuenta el hospital San Pablo de Coquimbo previo a la implementación del proceso.

- Recursos físicos: El hospital cuenta con unidad de almacenamiento de tejidos desde 2016, para uso de la especialidd de traumatología. Dicha unidad cuenta con espacio físico y la implementación necesaria para su funcionamiento, cuenta ade,ás con el premiso sanitario corrspondiente para su funcionamiento. Se cuenta con protocolo de procuramiento de huesos y de procuramiento de membrana amniótica.

- Recursos humanos: 3 enfermeras 44 horas y sistema de turno de llamado para emergencias $24 / 7$, todas ellas capacitadas en proceso de procuramiento y co contacto directo con las unidades de procuramiento a nivel central. El hospital cuenta ademas con turno de 24/7 de neurociirujano.

\section{- Establecer brechas y evaluación de soluciones adop-} tadas en base a la evidencia científica.

- Selección paciente y definición de proceso de consentimiento y coordinación. 
Para el hospital de Coquimbo se definió que el paciente candidato suceptible de guardar su plaqueta ósea es aquel paciente atendido por neurocirujano, el cual defina pudiese requerir una cranectomia descompresiva en el curso de su cirugía o la requiera como indicación inicial.

No se estableció límite de edad pues la literatura evidencia que es efectivo tanto en niños como adultos ${ }^{21}$.

Se estableció que en el mismo consentimiento informado establecido en el hospital (Anexo 1), se considerara el procuramiento de la plaqueta ósea del paciente, siendo el mismo neurocirujano encargado de informar a los familiares en este aspecto.

Si bien a los pacientes donantes se le exije una bateria de estudios serologicos, por corresponder a un autoinjerto estos se establecieron sólo como obigatorios para el $\mathrm{VIH}$ (Virus de Inmuno Deficiencia Humana), serología de Hepatitis
B y Hepatitis C. Esto se solicita para verificar si el paciente era portador antes del procedimiento, de modo tal que una infección no pueda asociarse a cobtaminación de la plaqueta.

Posterior a esto se debe comunicar con la unidad de procuramiento para que asista al procedimiento.

- Proceso de procuramiento en pabellón:

El procedimiento de extracción de la plaqueta debe ser bajo estricta técnica aséptica, la arsenalera la debe cubrir con compresaestéril hasta ser entregado al personal de procuramiento.

Se limpiará la mayor cantidad de sangre y se almacenará en triple bolsa estéril de 90 um de espesor.

- Traslado y conservación de la calota ósea:

El tejido procurado se trasdará a la unidad de almacenamiento de tejidos en un contenedor especial para almacenamiento.

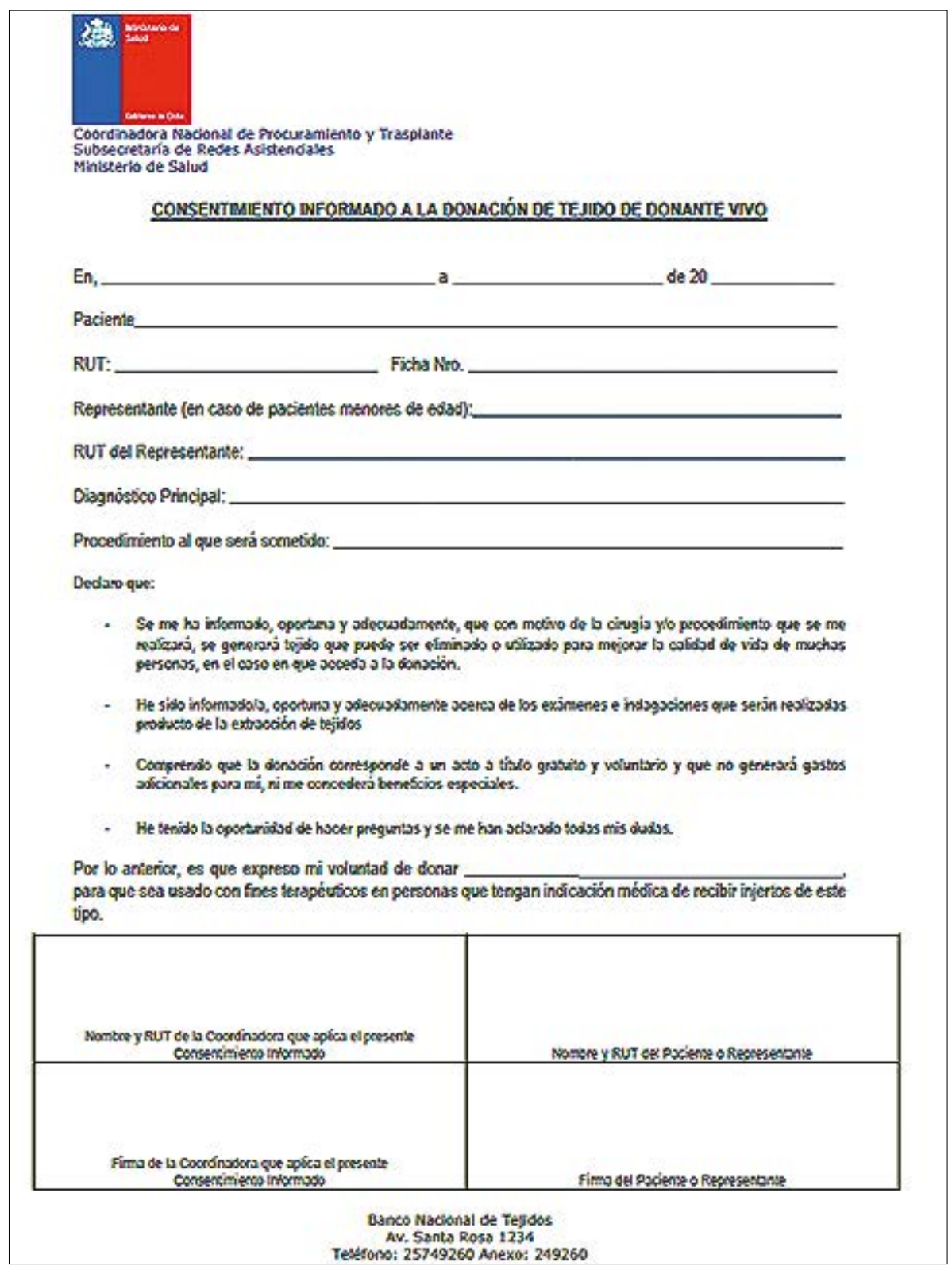


- Almacenamiento:

El tejido se mantendrá en cuarentena por periodo máximo de 5 años desde la fecha de procuramiento y será eliminadoo según normativa REAS (reglamento sobre manejo de residuos de establecimientos de atención de salud).

- Implante de autoinjerto óseo:

Cuando se solicite la craneoplastia del paciente, se debe coordinar el procedimiento de esterilización de la plaqueta ósea.

Para eso es necesario un procesamiento de los tejidos, se evaluará la carga microbiólogica.

Para la esterilización hay diferentes técnicas con gas de autoclave u óxido de etileno para prevenir la infección post operatoria $^{22}$. Actualmente, se recomienda la irradiación con rayos gamma, pues presenta los mejores resultados microbiológicos ${ }^{23}$.

La irradiación se realiza de acuerdo a un procedimiento estandarizado y conforme a los resultados de los análisis de las cargas microbianas realizadas en los tejidos ${ }^{24}$.

En Chile, para realizar este proceso las plaquetas óseas deben ser derivadas a la CCHEN (Comisión Chilena de Energía Nuclear).

\section{- Realización y sociabilización del protocolo}

Una vez elaborado el protocolo en conjunto con equipo de unidad de procuramiento, urgencia y subdirección médica, este es aprobado y establecido como protocolo del hospital a contar del 01 - 08 - 2018.

Posteriormente, se realiza la sociabilización del protocolo con todos los equipos involucrados.

\section{- Puesta en marcha del proceso:}

Se comienza con el procuramiento de plaquetas óseas en septiembre de 2018, durante este período hasta marzo de 2019, se han procurado 12 plaquetas óseas, 1 ha debido eliminarse por contaminación y 6 por fallecimiento del paciente y 3 se han reimplantado.

2. Valorización de proceso de procuramiento y reposición de plaqueta ósea autóloga en hospital San Pablo de Coquimbo

Al valorizar el costo del procedimiento desde el procuramiento hasta el implante de la plaqueta, se determino lo siguiente:

Se utilizará la infraestructura ya instalada para el almacenamiento.

Insumo para procuramiento: 10.000

Transporte a CCHEN: 216.000

Costo irradiación CCHEN: 25.000

Costo total del procedimiento: 251.000

\section{Descripción de los pacientes operados}

Durante el período de implementación a todos los pacientes cranectomizados se les ha procurado su plaqueta ósea. Con un total de 13 pacientes.

Se ha eliminado un total de 6 plaquetas. 1 por contaminación y 5 por fallecimiento de los pacientes.

A 3 pacientes han completado el proceso con la reinsta- lación de la plaqueta, los que se describen a continuación.

- A.P.F. Paciente de 3 años, victima de atropello, ingresa a hospital de Coquimbo en glasgow 3 con reflejos de tronco. TAC de ingreso con hematoma subdural izquierdo con efecto de masa. En intraoperatorio se encuentra paciente con cerebro edematoso impide recolocar plaqueta por lo que se cranectomiza hemisférico izquierdo. Posterior a eso se maneja por protocolo de TEC (traumatismo craneoencefalico) evolucionando con recuperación neurologica, activo hemiparesia severa derecha. Es dado de alta y se reoloca plaqueta ósea por cirugía electiva a los 2 meses post accidente. Cirugía de duración de 70 minutos, post operatorio de 48 horas. Hasta el momento del control se encontraba en buenas condicones buen resultado estético, sin complicaciones.

- I.V.F. Paciente de 2 años, caído de balcón, ingresa sedado anisocorico en glasgow 3 . TAC de ingreso múltiples lesiones supratentoriales, hematoma cortical parieto occipital izquierdo y hematoma yuxtadural de mismo lado. Se interviene por urgencia, se evidencia sangramiento intraoperatorio importante con paro intrapabellón. Paciente queda cranectomizado, se maneja en UCIP (Unidad de Cuidados Criticos Pediátrico), según protocolo de TEC, evolucionando con recuperación progresiva. Es dado de alta en buenas condiciones hemiparesia leve derecha, amaurosis bilteral. Se reinstala plaqueta a los 45 días posteriores en forma electiva, procedimiento sin incidentes, duración de 50 minutos de cirugía, post operatorio de 48 horas, al control buenas condicones, buen resultado estético.

- A.P.F. Paciente adulto, masculino de 37 años, ingresa posterior a caída desde muro en estado de ebriedad, al ingreso glasgow 7 con hematoma extradural izquierdo y contusiones fronto temporales derechas. Se interviene inicialmente de hematoma extradural y se maneja en UCI (Unidad Cuidados Intensivos) con monitoreo de PIC (presión intracraneana) según protocolo de TEC, evoluciona refractariamente a tratamiento por lo que se cranectomiza a las 24 horas posteriores. Se cranectomiza hemisférico derecho. Al control de alta se evidencia paciente con paresia mínima izquierda y tratorno cognitivo pero independiente para sus actividades de la vida diaria, indicandosele craneoplastia y solicitando inicio de proceso de esterilización de plaqueta. Se reinstala plaqueta a los 3 meses post operatorios. Sin complicaciones y buen resultado estético.

\section{Discusión}

La craneoplastia en los pacientes descomprimidos en Servicios de Neruocirugía es un problema constante, dado que ninguna de las alternativa de las disponibles es completamente segura y esta sujeta a complicaciones.

Los diferentes materiales actualmente disponibles para la reconstrucción craneal de tipo aloplásticos como el titanio, metilmetacrilato y la criptonita. El material ideal para esta reconstrucción debe tener como caracterísrica ser radiolúcido, mal conductor térmico, biocompatible, con potencial osteogénico, compatible con estudios de imagen, accesible, 
reistente y maleable. Por lo que, aún los laboratorios están trabajando en materiales innovadores para conseguirlo25,26.

El injerto autólogo presenta algunas ventajas importantes, como menor prevalencia de infección, exposición, rechazo y menor respuesta tisular, pero tienen como contarparte mórbida la reabsorción del tejido y morbilidad propia del sitio del donante. Por otro lado, el material aloplástico tiene la particularidad de ofrecer mayor resisitencia, nula reabsorción, menor dificultad para la remodelación, teniendo como inconveniente mayor prevalencia de infección, mayor reacción tisular, rechazo y costo mayor. En ambos se presenta la misma prevalencia de exposición ${ }^{27,28}$.

Considerando las condiciones actuales de nuestro centro, hemos definido que las craneoplastias se ofrecerá como primera posibilidad la colocación de hueso autólogo si se encuentra disponible y segunda opción malla de titanio o cemento que esl o que actulmente tenemos disponible.

Dado que los bancos de huesos son cada vez más frecuentes en los hospitales, es que se considera que la implementación de procuramiento de calota ósea es un procedimiento factible con costos no tan elevados y con seguridad, de modo tal de ofrecer la alternativa para reconstrucción en los pacientes.

Se debe tener presente que al ser una técnica nuevamente incorporada, debe ser riguroso los controles de calidad, vigilancia de efectos adversos y permanente revisión del proceso en cuestión en los agentes involucrados en su aplicación.

Es así como el hospital de Coquimbo es el primer hospital público chileno que tiene incorporado este procedimiento dentro de su catera de servicios, según los registros consultados en registros del MINSAL (Ministerio Salud Chile).

Al considerar los costos hay varios estudios que han evaluado costo efectividad del autoinjerto autologo por criopreservación, como la mejor alternativa para ofrecer en craneoplastias $^{18}$. Al analizar los costos asociados para implementar esta técnica en hospitales que ya cuentan con la infraestructura de banco de almacenamiento de tejidos, se encuentra que los costos son menores en comparación con lo utilizado actualmente en el hospital de Coquimbo que es la malla de titanio, la cual tiene un costo de 550.000 pesos en promedio. $Y$ hay mucha diferencia con las plaquetas a medida con metacrilato cuyo costo puede estar en los 2.000.000 pesos.

En la Tabla 1 se resume la comparación de las características de cada una de las alternativas de craneoplastias.

Considerando, la factibilidad de implementar el procuramiento de plaqueta ósea y con el costo antes mencionado, es que la alternativa de usar el hueso autologo por criopreservación como la primera alternativa para realizar craneoplastia dado que es la más costoefectiva de las disponibles hasta el momento en nuestro centro.

\section{Conclusiones}

La craneoplastia con hueso autólogo es una de la mejores alternativas para craneoplastia en pacientes neuroquirúrgios descomprimidos, con tasas de comlicaciones comparables a los otras alternativas terapeúticas.

La implementación del procuramiento de plaqueta ósea para craneoplastia autóloga por criopreservación es un proceso factible de realizar en un hospital de mediana complejidad y existiendo la posibilidsd de realizar parte del proceso a través de otras entidades, considerando las normativas vigentes.

Los costos asociados al proceso de procuramiento e implante, una vez establecido el banco de almacenamiento de huesos, son aceptables e incluso más económicos que el actualmente disponible en el hospital, dejandolo como la alternativa más costo-efectiva para estapatología.

\section{Referencias}

1. Aarabi B, Hesdorffer DC, Ahn Es, et al. Resultado después de la cranectomia descompresiva para lesiones graves del cerebro. $\mathrm{J}$ Neurosurg 2006; 104: 469-79.

2. Bell RS, Mossop CM, Dirks MS, Stephens FL, Mulligan L, Ecker $\mathrm{R}$, et al. Early decompressive craniectomy for severe pene-

\begin{tabular}{|c|c|c|c|}
\hline \multicolumn{4}{|c|}{ Tabla 1} \\
\hline Características & Plaqueta por autoinjerto & Aloinjerto de titanio & Aloinjerto por polímero \\
\hline Disponibilidad & $\begin{array}{l}\text { Requiere instalación de } \\
\text { banco de almacenamiento } \\
\text { de tejidos }\end{array}$ & $\begin{array}{l}\text { Sólo requiere adquisición de } \\
\text { malla de titanio }\end{array}$ & $\begin{array}{l}\text { Puede solicitarse su compra } \\
\text { a través de tomografía axial } \\
\text { con reconstrucción } 3 D \text { (tri } \\
\text { dimensional), de realizarla } \\
\text { en hospital requiere el } \\
\text { insumo y la impresora 3D }\end{array}$ \\
\hline Infecciones & Menores & Mayores & Mayores \\
\hline Rechazo mayor & Muy escaso & Mayor & Mayor \\
\hline Resultados estáticos & Buenos & Regulares & Muy buenos \\
\hline Período de craneoplastia & Hasta 6 meses & Indefinidos & Indefinidos \\
\hline Osteolísis & Mayor & Nula & Nula \\
\hline Capacidad de remodelación & Nula & Regular & Nula \\
\hline Costos & 251.000 & 550.000 & 2.000 .000 \\
\hline
\end{tabular}


trating and closed head injury during wartime. Neurosurgical focus. 2010 May; 28(5): E1. PubMed PMID: 20568925. Epub 2010/06/24. eng.

3. Caro-Osorio E, De la Garza-Ramos R, Martínez-Sánchez, et al. Cranioplasty with polymethylmethacrylate prostheses fabricated by hand using original bone flaps: techinacal note and surgical outcomes. Surg Neurol int, 2013, pág 136-139.

4. Carvi Y, Nievas MN, Höllerhage HG. Early combined cranioplasty and programmable shunt in patients with skull bone defects and CSF-circulation disorders. Neurol Res 28:139-144, 200612.

5. Chaturvedi J, Botta R, Prabhuraj AR, Shukla D, Bhat DI, Devi BI. Complications of cranioplasty after decompressive craniectomy for traumatic brain injury. Br J Neurosurg 30:264-268, 2016.

6. Liang W, Xiaofeng Y, Weiguo L, Gang S, Xuesheng Z, Fei C, et al. Cranioplasty of large cranial defect at an early stage after decompressive craniectomy performed for severe head trauma. J Craniofac Surg 18:526-532, 2007.

7. Quah BL, Low HL, Wilson MH, Bimpis A, Nga VD, Lwin S, et al. Is there an optimal time for performing cranioplasties? Results from a prospective multinational study. World Neurosurg 94:1317, 2016.

8. Khader BA, Towler MR. Materials and techniques used in cranioplasty fixation: A review. Mater Sci Eng C Mater Biol Appl 66: 315-322, 2016.

9. Arun Kumar KV, Singla NK, Gowda ME, Kumar D, Legha VS. Current Concepts in Restoring Acquired Cranial Defects. J Indian Prosthodont Soc 14:14-17, 2014.

10. Lemée JM, Petit D, Splingard M, Menei P. Autologous bone flap versus hydroxyapatite prosthesis in first intention in secondary cranioplasty after decompressive craniectomy: a French medicoeconomical study. Neurochirurgie 59:60-63, 2013.

11. Plum AW, Tatum SA. A comparison between autograft alone, bone cement, and demineralized bone matrix in cranioplasty. Laryngoscope 125:1322-1327, 2015.

12. Stefini $R$, Zanotti $B$, Nataloni $A$, Martinetti $R$, Scafuto M, Colasurdo $M$, et al. The efficacy of custom-made porous hydroxyapatite prostheses for cranioplasty: evaluation of postmarketing data on 2697 patients. J Appl Biomater Funct Mater 13:e136-e144, 2015.

13. Shaffrey ME, Persing JA, Shaffrey Cl. Craniofacial reconstruction in Apuzzo MLJ (ed): Brain surgery: Complication avoidance and management. New York, NY: Churchill Livingstone, pp13731398, 1993.

14. Lee BS, Min KS, Lee MS, Kim YG, Kim DH. Comparison with subcutaneous abdominal preservation and cryoconservation using autologous bone flap after deco.
15. Clune JE, Mulliken JB, Glowacki J, Arany PR, Kulungowski AM, Rogers GF, et al. Autologous cranial particulate bone graft: an experimental study of onlay cranioplasty. J Craniofac Surg 22:319- 323, 2011.

16. Son S, Park CW, Kim EY, Kim JM, Yoo CJ. Bone resorption of autologous cranioplasty following decompressive craniectomy in children: case report. J Korean Neurotraumatol Soc 5:118-123, 2009.

17. Bhaskar IP, Yusheng L, Zheng M, Lee GY. Autogenous skull flaps stored frozen for more than 6 months: do they remain viable? J Clin Neurosci 18:1690-1693, 2011.

18. Lethaus B, Bloebaum M, Koper D, Poort-Ter Laak M, Kessler P. Interval cranioplasty with patient-specific implants and autogenous bone grafts-success and cost analysis. J Craniomaxillofac Surg 42: 1948-1951, 2014.

19. Takeuchi H, Higashino $\mathrm{Y}$, Hosoda T, Yamada S, Arishima H, Kodera $\mathrm{T}$, et al. Long-term follow-up of cryopreservation with glycerol of autologous bone flaps for cranioplasty after decompressive craniectomy. Acta Neurochir (Wien) 158:571-575, 2016.

20. Kuleshova LL, Gouk SS, Hutmacher DW. Vitrification as a prospect for cryopreservation of tissue-engineered constructs. Biomaterials 28:1585-1596, 2007.

21. Bowers CA, Riva-Cambrin J, Hertzler DA, Walker ML. Risk factors and rates of bone flap resorption in pediatric patients after decompressive craniectomy for traumatic brain injury. $\mathrm{J}$ Neurosurg Pediatrics. 2013;11:526-32.

22. Son S, Park CW, Kim EY, Kim JM, Yoo CJ. Bone resorption of autologous cranioplasty following decompressive craniectomy in children: case report. J Korean Neurotraumatol Soc 5:118-123, 2009.

23. Endres S, Kratz M. Gamma irradiation. An effective procedure for bone banks, but does it make sense from an osteobiological perpective. J musculoskelet Neuronal Interact, 2009, 9(1):25-31.

24. Guía de Buenas Prácticas, ALABAT 2012.

25. Spetzger U, Vougioukas V, Schipper J. Materials and techniques for osseous skull reconstruction. Minim Invasive Ther Allied Technol. 2010;19(2):110-121.

26. Jaskolka MS, Olavarria G. Reconstruction of skull defects. Atlas Oral Maxillofac Surg Clin North Am. 2010;18(2):139-149.

27. Han SE, Lim SY, Pyon JK, et al. Aesthetic refinement of secondary cranioplasty using methylmethacrylate bone cements. Aesth Plast Surg. 2013;37(3):592-600.

28. Doumit GD, Meisier E, Sidaoui J, et al. The expansile properties of kryptonite relating to cranioplasty. J Craniofac Surg. 2014;25(3):880-883. 